\title{
Efectos genéticos en el crecimiento predestete de corderos Hampshire
}

\author{
Rodríguez-Almeida, F.A. ${ }^{\text {; }}$ Pérez-Camacho, C. ${ }^{\text {; }}$ Domínguez-Viveros, J. ${ }^{1 @}$ y de la Cruz-Colín, L. ${ }^{2}$
}

'Facultad de Zootecnia y Ecología. Universidad Autónoma de Chihuahua. Chihuahua. México.

${ }^{2}$ Instituto Nacional de Investigaciones Forestales, Agrícolas y Pecuarias. Pachuca. México.

Palabras ClaVe adicionales

Crecimiento predestete.

Heredabilidad.

Correlación genética.

Antagonismo genético.

Parámetros genéticos.

\section{RESUMEN}

Se analizó en corderos Hampshire el peso a 30 días (P30; 1072) y al destete (P75; 1750) de 10 rebaños, para desarrollar dos objetivos: evaluar la expresión de los efectos genéticos en el crecimiento predestete, y analizar las posibles implicaciones en el progreso genético. Los análisis fueron univariados (ANAU) y bivariados (ANAB), a través de modelos mixtos que incluyeron los efectos fijos de grupos contemporáneos y la covariable de edad de la madre; y como aleatorios, los efectos genéticos directos y maternos, la covarianza genética entre ellos, y los efectos maternos de ambiente permanente. Se estimó la heredabilidad directa $\left(\mathrm{h}^{2}\right)$ y materna $\left(\mathrm{m}^{2}\right)$, las correlaciones genéticas (rdm), y los valores genéticos (VG). Con correlaciones de rango (CR) en los VG se analizó la jerarquización de la población y de los sementales; además, se analizaron las tendencias genéticas (TG) de los VG. La h² estuvo en el intervalo de $0.14 \pm 0.03$ a $0.19 \pm 0.04$; la m² en ANAU fue de $0.08 \pm 0.04$ y $0.02 \pm 0.03$, con estimación en $A N A B$ de $0.22 \pm 0.05$ y $0.13 \pm 0.03$. La rdm del ANAU fue $+1 \pm 0.15$ en P30 y $-1 \pm 0.18$ en PD, cambiando en el ANAB a $-0.46 \pm 0.10$ y $-0.20 \pm 0.07$, respectivamente. El comportamiento de las TG en el ANAU estuvo asociada a la magnitud y el signo de las rdm; asimismo, las rdm del ANAU implicaron cambios en jerarquización de sementales, lo cual se vio reflejado en las TG y las CR.

\section{Genetic effects on pre-weaning growth of hampshire lambs}

\section{SUMMARY}

\section{AdDITIONAL KEYWORDS}

Preweaning growth.

Heritability.

Genetic correlation.

Genetic antagonism.

Genetic parameters.

\section{INFORMATION}

Cronología del artículo.

Recibido/Received: 29.03.2018

Aceptado/Accepted: 17.09.2018

On-line: 07.04.2019

Correspondencia a los autores/Contact e-mail:

jodominguez@uach.mx
The 30-day weight (P30; 1072) and the weaning (P75; 1750) of 10 flocks were analyzed in Hampshire lambs, to develop two objectives: to evaluate the expression of the genetic effects in the preweaning growth, and to analyze the possible implications in the genetic progress. The analyzes were univariate (ANAU) and bivariate (ANAB), through mixed models that included the fixed effects of contemporary groups and the covariable age of the mother; and as random, the direct and maternal genetic effects, the genetic covariance between them and the maternal effects of the permanent environment. Direct heritability (h2) and maternal (m2), genetic correlations (rdm), and genetic values (VG) were estimated. With the rank correlations (CR) in the VG, the ranking of the population and of the sire was analyzed; in addition, the ge netic trends (TG) of the VGs were analyzed. The $h 2$ was in the range of $0.14 \pm 0.03$ to $0.19 \pm 0.04$; the $\mathrm{m} 2$ in $A N A U$ was $0.08 \pm 0.04$ and $0.02 \pm 0.03$, with $A N A B$ the estimate was of $0.22 \pm 0.05$ and $0.13 \pm 0.03$. The rdm of $A N A U$ was $+1 \pm 0.15$ in $P 30$ and $-1 \pm 0.18$ in $P D$, changing in the $A N A B$ to $-0.46 \pm 0.10$ and $-0.20 \pm 0.07$, respectively. The behavior of the TG in the ANAU was associated to the magnitude and sign of the rdm; likewise, the rdm of the ANAU implied changes in the ranking of sires, which was reflected in the TG and the CRs.

\section{INTRODUCCIÓN}

El estudio del crecimiento de los rumiantes en Latinoamérica está tomando mucha relevancia especialmente en el bovino (Barbosa et al. 2017). En ovinos, el peso al destete es una de las principales características de interés económico, como criterio de selección tiene incidencia en la eficiencia y productividad del rebaño, si bien en esta especie cobran importancia económica otros objetivos, como la prolificidad (Menéndez-Buxadera et al. 2016). En el crecimiento predestete, el cordero tiene la tasa de crecimiento más alta de toda su vida, es una medida del potencial de crecimiento del cordero dado los efectos genéticos aditivos directos, y de las cualidades de la madre a través aspectos fisiológicos y 
Tabla I. Parámetros genético para peso ajustado a los $30 \mathrm{~d}(\mathrm{P} 30)$ y peso al destete (PD) ajustado a $75 \mathrm{~d}$ en ovinos Hampshire (Genetic parameters for weight set at $30 \mathrm{D}$ (P30) and weaning weight (PD) adjusted to 75 D in Hampshire sheep).

\begin{tabular}{|c|c|c|c|c|c|c|c|}
\hline \multicolumn{5}{|c|}{ Análisis bivariado } & \multicolumn{3}{|c|}{ Análisis univariado } \\
\hline & P30D & PDD & P30M & PDM & & P30D & P30M \\
\hline P30D & $0.18 \pm 0.03$ & & & & P30D & $0.19 \pm 0.04$ & \\
\hline PDD & $0.87 \pm 0.11$ & $0.14 \pm 0.03$ & & & P30M & $1.00 \pm 0.15$ & $0.08 \pm 0.04$ \\
\hline P30M & $-0.46 \pm 0.10$ & $-0.38 \pm 0.09$ & $0.22 \pm 0.05$ & & & & \\
\hline \multirow[t]{3}{*}{ PDM } & $-0.28 \pm 0.05$ & $-0.20 \pm 0.07$ & $0.98 \pm 0.13$ & $0.13 \pm 0.03$ & & PDD & PDM \\
\hline & & & & & PDD & $0.15 \pm 0.04$ & \\
\hline & & & & & PDM & $-1.00 \pm 0.18$ & $0.02 \pm 0.03$ \\
\hline
\end{tabular}

P30D y P30M = efectos genéticos directos y maternos para P30, respectivamente. PDD y PDM = efectos genéticos directos y maternos para PD, respectivamente. Sobre la diagonal son heredabilidades y bajo la diagonal son correlaciones genéticas.

etológico, así como la producción de leche, definidos como efectos genéticos aditivos maternos. En los modelos para analizar el peso al destete generalmente se incluyen los efectos genéticos directos y maternos, y la correlación genética entre ambos efectos (Willham, 1972; Bijma, 2006); con estimaciones negativas e intermedias (Boujenane y Kansari, 2002; Maniatis y Pollott, 2003), positivas (Abbasi et al., 2012) o nulas (Duguma et al., 2002). Sin embargo, cuando la correlación genética es negativa y cercana a la unidad, expresa un antagonismo entre ambos efectos, con posibles repercusiones en la selección con base en valores genéticos o de cría (Kariuki et al., 2010; Zishiri et al., 2014; David et al., 2015).

Los criadores de ovinos Hampshire en México implementaron un programa de mejora genética para variables de crecimiento y reproductivas con base en la selección a través de valores genéticos, y el uso común de sementales de referencia (de la Cruz-Colín et al., 2006; Ramírez-Tello et al., 2013). Este programa permitió utilizar animales seleccionados bajo un esquema de sementales de referencia, extendiendo las conexiones genéticas entre rebaños y conformando una base de datos de peso vivo distribuidos en el periodo predestete. El esquema de sementales de referencia fortalece la integridad y estructura del pedigrí, así como la conectividad genética a través de rebaños y de grupos contemporáneos. No obstante, en el crecimiento predestete, la expresión de los efectos genéticos directos y maternos, así como la correlación genética entre ambos presenta diferencias en función del avance de la lactancia (Kirkpatrick y Lande, 1989).
Con base en lo anterior, en el presente estudio se desarrollaron dos objetivos: para analizar la expresión de los efectos genéticos en el crecimiento predestete, se estimaron componentes de varianza y parámetros genéticos para peso a los 30 y 75 días de edad; y, para analizar las posibles implicaciones en el progreso genético, se evaluaron los efectos de las correlaciones genéticas en las tendencias genéticas y la jerarquización de sementales.

\section{MATERIALES Y MÉTODOS}

Se analizó la base de datos de diez rebaños ubicados en los estados de México e Hidalgo, con 1072 datos de peso ajustado a los $30 \mathrm{~d}$ (P30), y 1750 datos de peso al destete ajustado a los $75 \mathrm{~d}$ (PD) de edad. El peso promedio fue de $13.3 \pm 2.65 \mathrm{~kg}$ para $\mathrm{P} 30$ y $27.6 \pm 5.96$ $\mathrm{kg}$ para PD. El archivo de pedigrí quedo conformado por 2775 registros. Se realizaron análisis univariados (ANAU) y bivariados (ANAB) con un modelo mixto: $\mathbf{y}=\mathrm{X} \beta+\mathbf{Z} \mathbf{g}+\mathbf{M m}+\mathbf{W} \mathbf{p}+\mathbf{e}$; donde $\mathbf{y}$ es el vector de registros de P30 y PD; $\beta$ es el vector de efectos fijos, que incluyó los grupos contemporáneos definidos por las combinaciones de rebaño, año, época de nacimiento y grupo de pesada, sexo y número de corderos nacidos vivos, más la covariable (lineal y cuadrática) edad de la madre al parto; $g$ es el vector de valores genéticos aleatorios directos (P30D y PDD para P30 y PD); $m$ es el vector de valores genéticos aleatorios maternos (P30M y PDM para P30 y PD); $p$ es el vector de efectos aleatorios maternos de ambiente permanente; $\boldsymbol{e}$ es el vector de efectos residuales; $X, Z, M$ y $W$ son matrices

Tabla II. Correlaciones de rango, entre los valores genéticos para peso a los $30 \mathrm{~d}$ (P30) y peso al destete (PD) a través de los análisis univariados y bivariado (Correlations of rank, between the genetic values for weight at $30 \mathrm{D}(\mathrm{P} 30)$ and weaning weight (PD) through univariate and bivariate analyses).

\begin{tabular}{lccc}
\hline & Todos los animales & \multicolumn{2}{c}{ Sementales } \\
\cline { 3 - 5 } & & Prec. $\leq 0.6$ & Prec. $>0.6$ \\
\hline P30D & 0.47 & 0.47 & 0.81 \\
P30M & 0.36 & 0.37 & -0.29 \\
PDD & 0.86 & 0.86 & 0.85 \\
PDM & -0.17 & -0.03 & 0.19 \\
\hline
\end{tabular}

P30D y P30M = valores genéticos directos y maternos para P30, respectivamente. PDD y PDM = valores genéticos directos y maternos para PD, respectivamente. Prec. $=$ Precisión. 

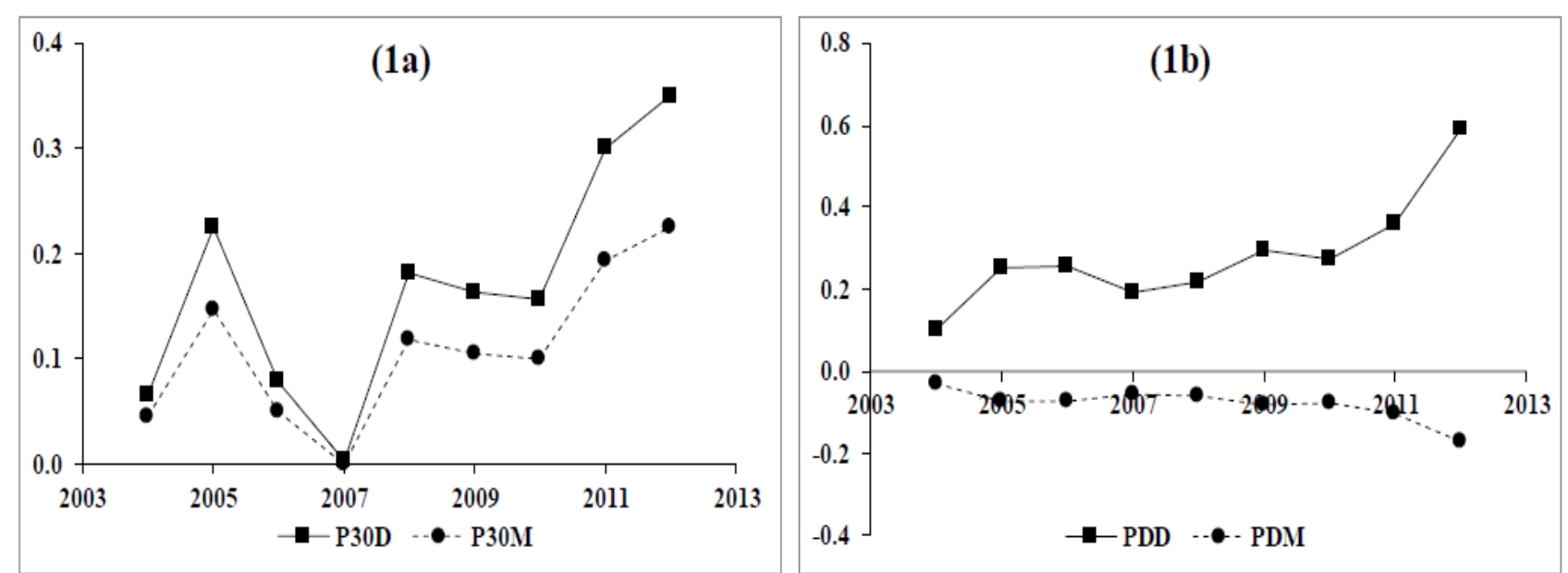

Figura 1. Tendencia de los valores genéticos directos (P30D - PDD) y maternos (P30M - PDM), producto del análisis univariado, para peso ajustado a los $30 \mathrm{~d}$ (1a) y peso al destete ajustado a $75 \mathrm{~d} \mathrm{(1b)} \mathrm{(Trends} \mathrm{of} \mathrm{direct} \mathrm{genetic}$ values (P30D - PDD) and maternal (P30M - PDM), product of univariate analysis, for weight adjusted to $30 \mathrm{D}(1 \mathrm{a})$ and weaning weight adjusted to $75 \mathrm{D}(1 \mathrm{~b})$.

de incidencia que asocian a los efectos en los vectores correspondientes con $y$. Los supuestos de los modelos evaluados fueron $E[y]=X \boldsymbol{\beta} ; E[\boldsymbol{g}]=E[\boldsymbol{m}]=E[\boldsymbol{p}]=E[\boldsymbol{e}]=\mathbf{0}$.

La estimación de componentes de (co)varianza y la predicción de valores genéticos se realizó con el programa para análisis multivariado, con máxima verosimilitud restringida y sin el uso de derivadas (MTDFREML; Boldmanet al., 1995); el criterio de convergencia, basado en la varianza del logaritmo de la función de verosimilitud, fue $1 \times 10^{-9}$. Se estimó para cada variable la heredabilidad de efectos genéticos directos $\left(\mathrm{h}^{2}\right)$ y maternos $\left(\mathrm{m}^{2}\right)$, y la correlación genética $\left(\mathrm{r}_{\mathrm{dm}}\right)$ entre ellos; entre P30 y PD las correlaciones entre efectos genéticos directos, efectos genéticos maternos, y efectos genéticos directos con efectos genéticos maternos $\left(\mathrm{r}_{\mathrm{dm} 2}\right)$. Para evaluar las implicaciones de las correlaciones genéticas, con las correlaciones de rango (CR) en los valores genéticos del ANAU vs ANAB se analizó el posible cambio en la jerarquización u ordenamiento de los valores de cría de toda la población, así como de los sementales en función de dos niveles de precisión $(\leq 0.6 \mathrm{y}>0.6)$; además, con el promedio de los valores genéticos en el año de nacimiento de los animales, se analizaron las tendencias a través del tiempo. Los análisis se realizaron con el programa para análisis estadísticos SAS (SAS, 2001).

\section{RESULTADOS Y DISCUSIÓN}

Las $\mathrm{h}^{2}$ estuvieron en el intervalo de $0.14 \pm 0.03$ a $0.19 \pm 0.04$ (Tabla I) en ambas variables, y sin cambios sustanciales $( \pm 0.01)$ del ANAU al ANAB; no obstante, las $\mathrm{m}^{2}$ en ANAU fueron de magnitud baja y cercanas a cero, con incrementos $( \pm 0.12)$ en el $A N A B, y$ valores máximos de $0.22 \pm 0.05$ en P30 (Tabla I). En la primera fase del crecimiento predestete, el cordero depende en gran medida de la habilidad materna y la producción de leche, por consiguiente, las diferencias en el crecimiento a través de corderos se pueden asociar a los efectos genéticos maternos; lo cual se refleja en los valores máximos de $\mathrm{m}^{2}$. En estudios similares Fadili et al. (2000), Safari et al. (2005) y Kariuki et al. (2010) reportaron estimaciones de $\mathrm{h}^{2}$ para P30 y PD en el intervalo de 0.11 a 0.34 para diversas razas de ovinos; asimismo,
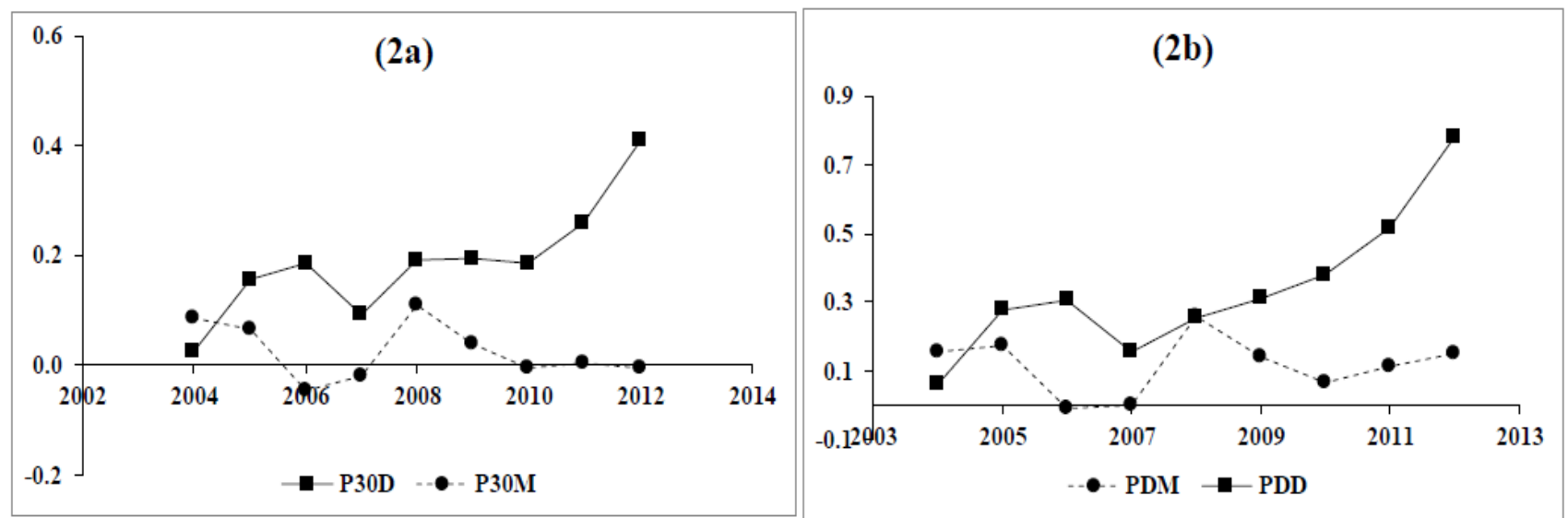

Figura 2. Tendencia de los valores genéticos directos (P30D - PDD) y maternos (P30M - PDM), producto del análisis bivariado, para peso ajustado a los $30 \mathrm{~d}(2 \mathrm{a})$ y peso al destete ajustado a $75 \mathrm{~d}(2 \mathrm{~b})$ (Tendency of direct genetic values (P30D - PDD) and maternal (P30M - PDM), product of the bivariate analysis, for weight adjusted to $30 \mathrm{D}(2 \mathrm{a})$ and weaning weight adjusted to $75 \mathrm{D}(2 \mathrm{~b})$. 
estimaciones de $\mathrm{h}^{2}$ menor o igual a 0.10 fueron reportadas por Boujenane y Kansari (2002) y Mandal et al. (2006). Además, Boujenane y Kansari (2002), Matika et al. (2003), Mandal et al. (2008) y Kariuki et al. (2010) publicaron estimaciones de $\mathrm{m}^{2}$ para P30 y PD en el intervalo de 0.05 a 0.20 , para diversas razas de ovinos nativas o locales, de los países donde se desarrolló el estudio.

Las $r_{d m}$ a partir del ANAU fueron sobre estimadas en ambos extremos, de $+1 \pm 0.15$ en P30 a $-1 \pm 0.18$ en PD; las $r_{d m}$ negativas y cercanas a la unidad, implica un antagonismo entre los genes para crecimiento y los genes para efectos maternos, y no permite avanzar en el mejoramiento con base en la selección de estos caracteres (María et al., 1993; Matika et al., 2003); por otro lado, estimaciones positivas y cercanas a la unidad, indica que desde el punto de vista genético son la misma característica (Safari et al., 2005). Resultados para $r_{\mathrm{dm}}$ negativos y cercanos a la unidad, fueron publicados por Fadili et al. (2000) y Neser et al. (2000); algunos autores (Robinson, 1996; Lee, 2002) han argumentado las razones porque estos valores pueden ser extremos, y otros cuestionan dichos valores con base en: a) la estructura de los datos no permite separar ambos efectos en el análisis (Clement et al., 2001; David et al., 2015); b) diferencias temporales en los ambientes cuando se expresan los efectos directos y los maternos (Kirkpatrick y Lande, 1989; Bijma, 2006); y, c) reducción en las varianzas de éstos efectos, debida a la selección realizada (Verrier et al., 1994; Lee, 2002).

Con el ANAB las $r_{d m}$ tuvieron mejores resultados y de manera conjunta con las $r_{d m 2}$, estuvieron en el intervalo de $-0.20 \pm 0.07$ a $-0.46 \pm 0.10$ (Tabla I); estos resultados coinciden en magnitud y signo a lo reportado por Boujenane y Kansari 2002, y difieren en signo a lo observado por Abbasi et al., (2012). Otros estudios han obtenido $\mathrm{r}_{\mathrm{dm}}$ en el intervalo de -0.50 a -0.80 (Mandal et al., 2006; Jafaroghli et al., 2010; Manzanilla et al., 2012). Las causas $u$ origen de las $r_{d m}$ negativas se ha abordado por diversos autores (Lee, 2002; Safari et al., 2005; Bijma, 2006); con $\mathrm{r}_{\mathrm{dm}}$ negativas y de mediana magnitud se pueden implementar programas de mejoramiento genético, con el objetivo de avanzar en ambas variables. Otra suposición sobre las $r_{\mathrm{dm}}$ negativas y su antagonismo genético, se puede atribuir a la curva de crecimiento de esta población Hampshire, la cual es de tipo sigmoide (Canul-Santos et al., 2014) y el punto de inflexión con sus implicaciones en el cambio de la tasa de crecimiento se ubica a los $68 \mathrm{~d}$, dentro del periodo predestete.

El comportamiento de los valores genéticos a través del tiempo (Figuras 1 y 2) está asociado a la trascendencia de la $r_{d m}$ en la respuesta a la selección. A partir del ANAU, en PD se observó un comportamiento (Figura 1b) muy diferente entre PDD y PDM, como consecuencia de la magnitud y el signo de la $\mathrm{r}_{\mathrm{dm}}(-1)$; asimismo, en P30 el P30D y P30M (Figura 1a) tienen una tendencia asociada a la $r_{d m}(+1)$. Estos resultados muestran las implicaciones y repercusiones de las correlaciones genéticas sobre la respuesta a la selección. A partir del ANAB, las tendencias genéticas presentan un comportamiento menos radical; lo observado en la Figuras $\mathbf{2 a}$ y $\mathbf{2 b}$ se atribuye a la selección realizada para efectos genéticos directos, desatendiendo los efectos genéticos maternos. Implementar esquemas de selección para efectos genéticos maternos, no tendría implicaciones drásticas sobre los efectos genéticos directos, dado la magnitud y el signo de las $r_{\mathrm{dm}}$.

La CR en la población completa fluctúo de 0.36 a 0.86 en P30D, P30M y PDD (Tabla II). Esto no implica repercusión en la jerarquización u ordenamiento de los animales por medio de los valores genéticos predichos con el ANAU vs ANAB; no obstante, para PDM la CR fue negativa y de baja magnitud (-0.17), señalando posibles cambios en el ordenamiento de algunos animales por el hecho del análisis que genera los valores genéticos. Las $r_{\mathrm{dm}}$ altas de los ANAU pueden afectar los valores genéticos predichos, sobre todo en los animales extremos; posteriormente, con el ANAB los rangos y los animales extremos tienden a disminuir con posibles implicaciones en la jerarquización. Las CR en los sementales con precisión igual o menor a 0.6 no indican un posible cambio en el ordenamiento; a diferencia de los sementales con precisión mayor a 0.6 en P30M, donde se obtuvo una correlación negativa con posibles implicaciones en el ordenamiento de los sementales. La precisión en la predicción de valores genéticos se debe a la cantidad de información genealógica y productiva analizada, y estos resultados se pueden atribuir a las ventajas o diferencias del ANAB vs el ANAU.

\section{CONCLUSIONES}

Las correlaciones genéticas cercanas a la unidad, producto del análisis univariado, tienen implicaciones en la jerarquización de sementales, lo cual se vio reflejado en las tendencias genéticas y en las correlaciones de rango.

Con el análisis bivariado, las correlaciones genéticas estuvieron en el intervalo de $-0.20 \pm 0.07$ a $-0.46 \pm 0.10$; con esta magnitud en las correlaciones genéticas, se pueden diseñar esquemas de selección para avanzar en ambas características.

\section{BIBLIOGRAFÍA}

Abbasi, MA, Abdollahi-Arpanahi, R, Maghsoudi, A, Torshizi, RV, \& Nejati-Javaremi, A 2012, 'Evaluation of models for estimation of genetic parameters and maternal effects for early growth traits of Iranian Baluchi sheep' Small Ruminant Research, vol. 104, pp. 62-69.

Barbosa, ACB, Carneiro, PLS, Rezende, MPG, Ramos, IO, Martins F \& Malhado, CHM 2017, 'Parâmetros genéticos para características de crescimento e reprodutivas em bovinos Nelore no Brasil', Archivos de Zootecnia, vol. 66, pp. 447-450.

Bijma, P 2006, 'Estimating maternal genetic effects in livestock', Journal of Animal Science, vol. 84, pp. 800-806.

Boldman, KG, Kriese, LA, Van Vleck, LD, Van Tassell, CP, \& Kachman, SD 1995. 'A manual for use of MTDFREML. A set of programs to obtain estimates of variances and covariances (Draft)', USDA, ARS. $114 \mathrm{p}$.

Boujenane, I \& Kansari, J 2002, 'Estimates of (co)variance due to direct and maternal effects for body weights in Timahdite sheep', Animal Science, vol. 74, pp. 409-414.

Canul-Santos E, Domínguez-Viveros, J, Rodríguez-Almeida, FA, OrtegaGutiérrez, JA \& Callejas-Juárez, N 2014, 'Evaluación de modelos no lineales para caracterizar el crecimiento de siete razas de ovinos en México', En: Memoria de la XLI Reunión anual de la Asociación Mexicana de Producción Animal, México, 648 pp. 
Clement, V, Bibé, B, Verrier, E, Elsen, JM, Manfredi, E, Bouix, J \& Hanocq, E 2001, 'Simulation analysis to test the influence of model adequacy and data structure on the estimation of genetic parameters', Genetic Selection Evolution, vol. 33, pp. 369-395.

David, I, Bouviers, F, Banville, M, Canario, L, Flatres-Grall, L, Balmisse, E \& Garreau H 2015, 'The direct-maternal genetic correlation has little impact on genetic evaluations', Journal of Animal Science, vol. 93, pp. 5639-5647.

de la Cruz-Colín, L, Torres-Hernández, G, Núñez-Domínguez, R \& Becerril-Pérez CM 2006, 'Evaluación de características productivas de corderos Hampshire, Dorset y Suffolk en pruebas de comportamiento en Hidalgo, México', Agrociencia, vol. 40, pp. 59-69.

Duguma, G, Schoeman, SJ, Cloete, SWP \& Jordaan, GF 2002, 'Genetic parameter estimates of early growth traits in the Tygerhoek Merino sheep', South African Journal of Animal Science, vol. 32, pp. 66-75.

Fadili EM, Michaux, C, Detilleux, J \& Leroy PL 2000, 'Genetic parameters for growth traits of the Moroccan Timahdit breed of sheep', Small Ruminant Research, vol. 37, pp. 203-208.

Jafaroghli, M, Rashidi, A, Mokhtari, MS \& Shadparvar AA 2010, '(Co) Variance components and genetic parameter estimates for growth traits in Moghani sheep', Small Ruminant Research, vol. 91, pp. 170-77.

Kariuki, CM, Ilatsia, ED, Kosgey, IS \& Kahi, AK 2010, 'Direct and maternal (co)variance components, genetic parameters and annual trends for growth traits of Dorper sheep in semi-arid Kenya', Tropical Animal Health and Production, vol. 42, pp. 473-481.

Kirkpatrick, M \&Lande R 1989, 'The evolution of maternal characters', Evolution, vol. 43, pp. 485-503.

Lee, C 2002, 'On the negative estimates of direct and maternal genetic correlation - a review', Asian-Australas Journal of Animal Science, vol. 15, pp. 1222-1226.

Neser, FWC, Erasmus, GJ \& van Wyk JB 2000, 'Genetic studies on the South African Mutton Merino: growth traits', South African Journal of Animal Science, vol. 30, pp. 172-177.

Mandal, A, Neser, FWC, Rout PK, Roy, R \& Notter DR 2006, 'Estimation of direct and maternal (co)variance components for pre-weaning growth traits in Muzaffarnagari sheep', Livestock Science, vol. 99, pp. 79-89.

Mandal, A, Roy, R \& Rout PK 2008, 'Direct and maternal effects for body measurements at birth and weaning in Muzaffarnagari sheep of India', Small Ruminant Research, vol. 75, pp. 123-127.

Maniatis, N \& Pollott GE 2003, 'The impact of data structure on genetic (co)variance components of early growth in sheep, estimated using an animal model with maternal effects', Journal of Animal Science, vol. 81, pp. 01-108.

Manzanilla PCI, Torres VJA, BorrayoZA, Ríos UA, Baeza RJJ, Martínez VG, Vega MVE \& Montaño BM 2012, 'Estimación de parámetros genéticos para características de crecimiento en borregos Katahdin usando diferentes modelos', Revista Mexicana de Ciencias Pecuarias, vol. 3, pp. 487-500

María, GA, Boldman, KG \& Van Vleck LD 1993, 'Estimates of variances due to direct and maternal effects for growth traits of Romanov sheep', Journal of Animal Science, vol. 71, pp. 845-849.

Matika, O, van Wyk, JB, Erasmus, GJ \& Baker, RL 2003, 'Genetic parameter estimates in Sabi sheep', Livestock Production Science, vol. 79, pp. 17-28.

Menéndez-Buxadera, A, Medina, C, Gómez, J, Barajas, F \& Molina A 2016, 'Parámetros genéticos de la prolificidad y la supervivencia hasta el sacrificio en corderos de raza Merina', Archivos de Zootecnia, vol. 65, pp. 291-296.

Ramírez-Tello, JA, Torres-Hernández, G, de la Cruz-Colín, L, OchoaCordero, MA \& Suárez-Espinosa, J 2013, 'Evaluación de factores ambientales que influyen en características de crecimiento del nacimiento al destete de corderos Hampshire', Revista Mexicana de Ciencias Pecuarias, vol. 4, pp. 117-125.

Robinson, DL 1996, 'Models which might explain negative correlations between direct and maternal genetic effects', Livestock Production Science, vol. 45, pp. 111-122.

Safari, E, Fogarty, NM \& Gilmour AR 2005, 'A review of genetic parameter estimates for wool, growth, meat and reproduction traits in sheep', Livestock Production Science, vol. 92, pp. 271-289.

SAS 2001, SAS User's Guide: Statistics (Release 9.0), Cary NC, USA: SAS Inst. Inc.

Verrier, E, Colleau JJ \& Foulley, JL 1994, 'Genetic variability when selecting on the animal model BLUP', In: Proceedings of the $5^{\text {th }}$ World Congress on the Genetics Applied to Livestock Production, Canada, vol. 19, pp. 139-142.

Willham, RL 1972, 'The role of maternal effects in animal breeding: III. Biometrical aspects of maternal effects in animals', Journal of Animal Science, vol. 35, pp. 1288-1293.

Zishiri, OT, Cloete, SWP, Olivier, JJ \& Dzama, K 2014, 'Genetic parameters for live weight traits in South African terminal sire sheep breeds', Small Ruminant Research, vol. 116, pp. 118-125. 